\title{
Local Sampled-Data Control of the Glucose-Insulin System
}

\author{
P. Pepe \\ P. Palumbo \\ S. Panunzi \\ A. De Gaetano
}

\begin{abstract}
In this work we consider the local sampleddata stabilization of human plasma glycemia. It is proved theoretically that the implementation by sampling and holding, for suitable small sampling period, of a state feedback which is shown in the literature to yield local stabilization when applied in a continuous time basis, yields local practical stabilization, with arbitrarily small final target ball. The model of the system is given by a nonlinear retarded functional differential equation and the above state feedback is provided by standard tools of differential geometry for time-delay systems. The proposed theoretical result proves an important property for the digital implementation of the controller, which has been shown in past literature to perform very well when checked in closed-loop with well known computer simulators of diabetic patients approved by the Food and Drug Administration as a substitute of animal trials.
\end{abstract}

\section{INTRODUCTION}

The problem of stabilization of systems described by ordinary differential equations by means of sampled-data control laws has been studied in many papers and many approaches are available in the literature in both the linear case (see, for instance, [5], [6], [7], [19], [37], [38]) and the nonlinear case (see, for instance, [10], [18], [22], [23], [25], [33], [35], [36], [39]). The sampled-data stabilization of nonlinear systems with delays in the input/output channels has been investigated in [13]. As far as systems described by retarded functional differential equations, an approach based on the notion of stabilization in the sample-and-hold sense, introduced in [3] (see also [2]), is proposed in [32], [34].

In this work we deal with an application of the methodology of the sample-and-hold stabilization. The problem at hand is the regulation of plasma glycemia at a desired level. We prove that it is possible to locally stabilize, in the sampleand-hold sense, the glucose-insulin system as described in the literature by retarded functional differential equations (see [26], [31] and references therein). Many results are nowadays available in the literature, mainly addressing glucose control problems on Type 1 diabetic patients, (see, for instance, [1], [4], [12], [16], [20], [30]). However, most of the proposed closed-loop control laws are designed in the continuous-time basis or, even when the discrete-time framework is preferred to cope with technological devices providing glucose measurements at sampling instants, very

This work has been partially supported by BIOMATLAB, Institute of Systems Analysis and Informatics, Italy.

P. Pepe is with the Department of Information Engineering, Computer Science and Mathematics, Center of Excellence DEWS, University of L'Aquila, 67100 L'Aquila, Italy, pierdomenico.pepe@univaq.it.

P. Palumbo, S. Panunzi, A. De Gaetano are with the Institute of Systems Analysis and Informatics, Italian National Research Council, Rome, Italy, pasquale.palumbo@iasi.cnr.rm.it, simona.panunzi@iasi.cnr.rm.it, andrea.degaetano@iasi.cnr.rm.it few theoretical results are available for their sampled-data implementation onto the physical continuous-time model. In [27], a sampled-data control law is proposed and stabilization is proved for just a suitable, discrete time approximation of the original continuous time model. No theoretical results are there given for the glucose-insulin system described by retarded functional differential equations. Here, instead, we provide local stabilization results for the closed-loop system, without any approximation, and in continuous time. Stabilization in the sample-and-hold sense is a practical stabilization with arbitrary small final target (i.e. arbitrary small neighborhood of the origin). A state feedback (continuous or not) is said to be a local stabilizer in the sample-and-hold sense if, for a suitable initial ball and an arbitrary small ball of the origin, there exists a suitable small sampling period such that the feedback control law obtained by sampling and holding the above state feedback, with the given sampling period, keeps uniformly bounded all the trajectories starting in any point of the initial ball and, moreover, drives all such trajectories into the small ball, uniformly in a maximum finite time, keeping them in, thereafter. Here, it is proved theoretically that the implementation by sampling and holding, for suitable small sampling period, of a given state feedback designed in the continuous time basis, which is shown in the literature to yield local stabilization (see [28]), provides local stabilization in the sample-and-hold sense. This result is expected, nevertheless its non trivial theoretical proof allows us to add a further important property to this controller proposed in the literature by the authors, which has been shown to perform very well when checked in closed-loop on a population of virtual patients modeled by means of the computer simulator in [17], accepted by the Food and Drug Administration (FDA) as a substitute to animal trials for the preclinical testing of control strategies in artificial pancreas (see [30]). The proof is obtained with the assumption of availability of the full state, that is, of insulin and glucose measurements, at sampling times. We believe that this theoretical proof provides a nice further property of the controller proposed in the literature, in the line of a safe use in practice. Indeed, in practice, measurements are available only at sampling times, as well as the control law is applied by means of digital devices.

The results provided here rely upon the paper [32], on the stabilization in the sample-and-hold sense of nonlinear retarded systems.

\section{Notations}

$R$ denotes the set of real numbers, $R^{+}$denotes the set of non negative reals $[0,+\infty), R^{\star}$ denotes the extended positive 
real line $[0,+\infty]$. The symbol $|\cdot|$ stands for the Euclidean norm of a real vector, or the induced Euclidean norm of a matrix. The essential supremum norm of an essentially bounded function is indicated with the symbol $\|\cdot\|_{\infty}$. For a positive integer $n$, for a positive real $\Delta$ (maximum involved time-delay), $\mathcal{C}$ and $W^{1, \infty}$ denote the space of the continuous functions mapping $[-\Delta, 0]$ into $R^{n}$ and the space of the absolutely continuous functions, with essentially bounded derivative, mapping $[-\Delta, 0]$ into $R^{n}$, respectively. A Lebesgue measurable function $w:[-\Delta, 0] \rightarrow R^{n}$ is said to be essentially bounded if ess $\sup _{t \in[-\Delta, 0]}|w(t)|<\infty$, where ess $\sup _{t \in[-\Delta, 0]}|w(t)|=\inf \left\{a \in R^{\star}: \lambda(\{t \in\right.$ $[-\Delta, 0]:|w(t)|>a\})=0\}, \lambda$ denoting the Lebesgue measure. For a positive real $p$, for $\phi \in \mathcal{C}, \mathcal{C}_{p}(\phi)=\{\psi \in$ $\left.\mathcal{C}:\|\psi-\phi\|_{\infty} \leq p\right\}$. The symbol $\mathcal{C}_{p}$ denotes $\mathcal{C}_{p}(0)$. For a continuous function $x:[-\Delta, c) \rightarrow R^{n}$, with $0<c \leq+\infty$, for any real $t \in[0, c), x_{t}$ is the function in $\mathcal{C}$ defined as $x_{t}(\tau)=x(t+\tau), \tau \in[-\Delta, 0]$. For a positive integer $n$, a positive real $q, B_{q}$ denotes the set of vectors $z \in R^{n}$, satisfying $|z| \leq q$. For given positive integers $n, m$, a map $f: \mathcal{C} \times R^{m} \rightarrow R^{n}$ is said to be Lipschitz on bounded sets if, for any positive real $q$, there exists a positive real $L_{q}$ such that, for any $\phi_{i} \in \mathcal{C}_{q}, u_{i} \in B_{q}, i=1,2$, the inequality holds $\left|f\left(\phi_{1}, u_{1}\right)-f\left(\phi_{2}, u_{2}\right)\right| \leq L_{q}\left(\left\|\phi_{1}-\phi_{2}\right\|_{\infty}+\left|u_{1}-u_{2}\right|\right)$. Let us here recall that a continuous function $\gamma_{R}^{+} \rightarrow R^{+}$is said to be of class $\mathcal{K}_{\infty}$ if it is zero at zero, strictly increasing, and unbounded. Throughout the paper, RFDE stands for Retarded Functional Differential Equation.

\section{Preliminary Results on Nonlinear Retarded SYSTEMS}

We report here for the reader's convenience some notions and results in [32], which will be used in next section devoted to the glucose-insulin system. Let us consider the system described by the following RFDE (see [11], [15])

$$
\begin{aligned}
& \dot{x}(t)=f\left(x_{t}, u(t)\right), \quad t \geq 0, \text { a.e., } \\
& x(\tau)=x_{0}(\tau), \quad \tau \in[-\Delta, 0], \quad x_{0} \in \mathcal{C},
\end{aligned}
$$

where: $x(t) \in R^{n}, n$ is a positive integer; $\Delta$ is a non-negative integer, the maximum involved time-delay; $x_{t} \in \mathcal{C} ; f$ is a map from $\mathcal{C} \times R^{m}$ to $R^{n}$, Lipschitz on bounded sets; $m$ is a positive integer; $u(t) \in U$ is a measurable signal, $U \subset R^{m}$ is a compact set containing the origin in the interior. We assume that $f(0,0)=0$. The equation (1) admits a locally absolutely continuous solution in a maximal time interval $[0, b)$, with $0<b \leq+\infty$ (see [11]). We introduce here the following assumption.

Assumption 1: (see [32]) The initial state belongs to $W^{1, \infty}$ and there exists a positive real $q$ such that ess $\sup _{\tau \in[-\Delta, 0]}\left|\frac{d x_{0}}{d \tau}\right| \leq q$.

We recall here the notion of partition of $[0,+\infty)$ (see [3], [2]).

Definition 1: ([3], [2], [32]) A partition

$$
\pi=\left\{t_{i}, i=0,1, \ldots\right\}
$$

of $[0,+\infty)$ is a countable, strictly increasing sequence $t_{i}$, with $t_{0}=0$, such that $t_{i} \rightarrow+\infty$ as $i \rightarrow+\infty$. The diameter of $\pi$, denoted $\operatorname{diam}(\pi)$, is defined as $\sup _{i \geq 0} t_{i+1}-t_{i}$. The dwell-time of $\pi$, denoted $d w e l l(\pi)$, is defined as $\inf _{i \geq 0} t_{i+1}-t_{i}$. For any positive reals $a \in(0,1], b>0$, $\pi_{a, b}$ is any partition $\pi$ with $a b \leq \operatorname{dwell}(\pi) \leq \operatorname{diam}(\pi) \leq b$.

Notice, in Definition 1, that $\pi_{1, \delta}$ is the partition with dwelltime equal to the diameter $\delta$ (i.e., the partition related to uniform sampling).

Definition 2: ([2], [3], [32]) Let $Q$ be a positive real. We say that a feedback $F: \mathcal{C}_{Q} \rightarrow U$ (continuous or not) stabilizes the system described by (1) in the sample-andhold sense, in $\mathcal{C}_{Q}$, if, for every positive reals $r, R, 0<r<$ $R \leq Q, a \in(0,1]$, there exist a positive real $\delta$ depending upon $r, R, q$ and $\Delta$, a positive real $T$, depending upon $r, R$, $q, \Delta$ and $a$, and a positive real $E$, depending upon $R$ and $\Delta$, such that, for any partition $\pi_{a, \delta}=\left\{t_{i}, i=0,1, \ldots\right\}$, for any initial state $x_{0} \in \mathcal{C}_{R}$, the solution corresponding to $x_{0}$ and to the sampled-data feedback control law

$$
u(t)=F\left(x_{t_{k}}\right), \quad t_{k} \leq t<t_{k+1}, \quad k=0,1, \ldots,
$$

exists $\forall t \geq 0$ and, furthermore, satisfies:

$$
x_{t} \in \mathcal{C}_{E}, \forall t \geq 0 ; \quad x_{t} \in \mathcal{C}_{r}, \forall t \geq T .
$$

Theorem 1: (see [32]) Let there exist a diffeomorphism $\Psi: \Omega_{x} \rightarrow \Omega_{z}$, with $\Omega_{x}, \Omega_{z} \subset R^{n}$ open, bounded neighborhoods of the origin, functions $\underline{\gamma}_{\psi}, \bar{\gamma}_{\psi}$, of class $\mathcal{K}_{\infty}$, a Hurwitz matrix $F \in R^{n \times n}$, a positive real $S$, a Lipschitz feedback $k: \mathcal{C}_{S} \rightarrow U$, zero at zero, such that: $\Omega_{x} \cap B_{S}=B_{S}$ (i.e., $\Omega_{x}$ contains the ball of the origin with radius $S$ );

$$
\begin{gathered}
\underline{\gamma}_{\psi}(|x|) \leq|\Psi(x)| \leq \bar{\gamma}_{\psi}(|x|), \quad \forall x \in \Omega_{x} \\
\left.\frac{\partial \Psi(x)}{\partial x}\right|_{x=\phi(0)} f(\phi, k(\phi))=F \Psi(\phi(0)), \quad \forall \phi \in \mathcal{C}_{S} .
\end{gathered}
$$

Then, there exists a positive real $Q<S$ such that the feedback $k: \mathcal{C}_{S} \rightarrow U$ stabilizes in the sample-and-hold sense, in $\mathcal{C}_{Q}$, the system described by (1).

\section{Stabilization By SAMPLING AND HOLding OF ThE GLUCOSE-INSULIN SYSTEM}

We show here that Theorem 1 can be successfully applied to the glucose-insulin system. Let us consider the following RFDE, mathematical model of the glucose-insulin system (see [26], [31])

$$
\begin{aligned}
& \frac{d G(t)}{d t}=-K_{x g i} G(t) I(t)+\frac{T_{g h}}{V_{G}} \\
& \frac{d I(t)}{d t}=-K_{x i} I(t)+\frac{T_{i G \max }}{V_{I}} h\left(G\left(t-\tau_{g}\right)\right)+v(t), \\
& G(\tau)=G_{0}, \quad I(\tau)=I_{0}, \quad \tau \in\left[-\tau_{g}, 0\right],
\end{aligned}
$$

where $G(t)$ (measurement unit $[\mathrm{mM}]$ ) is the plasma glycemia, $I(t)$ (measurement unit $[\mathrm{pM}]$ ) is the insulinemia, $t \geq 0$, and $G_{0}, I_{0}$ are related initial values. As far as the model parameters are concerned: 
- $K_{x g i},\left[\min ^{-1} \mathrm{pM}^{-1}\right]$, is the rate of glucose uptake by tissues (insulin-dependent) per pM of plasma insulin concentration;

- $T_{g h},\left[\mathrm{~min}^{-1}(\mathrm{mmol} / \mathrm{kgBW})\right]$, is the net balance between hepatic glucose output and insulin-independent zeroorder glucose tissue uptake (mainly by the brain);

- $V_{G},[\mathrm{~L} / \mathrm{kgBW}]$, is the apparent distribution volume for glucose;

- $K_{x i},\left[\mathrm{~min}^{-1}\right]$, is the apparent first-order disappearance rate constant for insulin;

- $T_{i \text { Gmax }},\left[\mathrm{min}^{-1}(\mathrm{pmol} / \mathrm{kgBW})\right]$, is the maximal rate of second-phase insulin release;

- $V_{I},[\mathrm{~L} / \mathrm{kgBW}]$, is the apparent distribution volume for insulin;

- $\tau_{g}$, [min], is the apparent delay with which the pancreas varies secondary insulin release in response to varying plasma glucose concentrations.

The nonlinear map $h(\cdot)$ models the endogenous pancreatic insulin delivery rate as $h(G)=\frac{\left(\frac{G}{G^{*}}\right)^{\gamma}}{1+\left(\frac{G}{G^{*}}\right)^{\gamma}}$, where $\gamma$ is the progressivity with which the pancreas reacts to circulating glucose concentrations and $G^{*}$ is the glycemia at which the insulin release is half of its maximal rate. The control input, $v(t)$ (measurement unit $[p M / m i n]$ ), is the exogenous intra-venous insulin delivery rate. Let $G_{r e f}$ be a positive constant, chosen as a reference level of glycemia instead of a hyperglycemic basal level $G_{b}>G_{r e f}\left(G_{b}\right.$ is the steady state value of glycemia when no input is applied, see Section IV). Let $I_{r e f}$ and $v_{r e f}$ be the positive reals such that $\left(G_{r e f}, I_{r e f}\right)$ is an equilibrium point for the system described by (6), forced by the constant input $v(t)=v_{\text {ref }}$ (see [29]). The positive reals $I_{r e f}$ and $v_{r e f}$ are obtained as solutions of the following algebraic equations

$$
\begin{aligned}
& -K_{x g i} G_{r e f} I_{r e f}+\frac{T_{g h}}{V_{G}}=0, \\
& -K_{x i} I_{r e f}+\frac{T_{i G m a x}}{V_{I}} h\left(G_{r e f}\right)+v_{r e f}=0
\end{aligned}
$$

The RFDE (6) can be rewritten with the new variables $x(t)=\left[\begin{array}{c}G(t)-G_{r e f} \\ I(t)-I_{\text {ref }}\end{array}\right]$ and with the new input $u(t)=$ $v(t)-v_{\text {ref }}$, as follows

$$
\begin{aligned}
& \frac{d x_{1}(t)}{d t}=-K_{x g i}\left(x_{1}(t)+G_{r e f}\right)\left(x_{2}(t)+I_{r e f}\right)+\frac{T_{g h}}{V_{G}}, \\
& \frac{d x_{2}(t)}{d t}=-K_{x i}\left(x_{2}(t)+I_{r e f}\right) \\
& +\frac{T_{i G m a x}}{V_{I}} h\left(x_{1}\left(t-\tau_{g}\right)+G_{r e f}\right)+v_{r e f}+u(t), \\
& x_{1}(\tau)=G_{0}-G_{r e f}, x_{2}(\tau)=I_{0}-I_{r e f}, \tau \in\left[-\tau_{g}, 0\right],
\end{aligned}
$$

Since the exogenous intra-venous insulin delivery rate cannot be negative, we have that the input $v(t)$ in (6) belongs to the following compact set $\bar{V}=\left[0, v_{\max }\right]$, where $v_{\max }>v_{\text {ref }}$ is a suitable positive bound due to technological as well as to physiological constraints (see [30]). It follows that $u(t)$ in
(8) belongs to the set $U=\left[-v_{r e f}, v_{\max }-v_{r e f}\right]$. The system described by (8) can be rewritten in the form of (1). Indeed, in this case, define $f: \mathcal{C} \times R \rightarrow U$, for $\phi=\left[\begin{array}{l}\phi_{1} \\ \phi_{2}\end{array}\right] \in \mathcal{C}$, $\phi_{i}(\tau) \in R, \tau \in\left[-\tau_{g}, 0\right], u \in U$, as

$$
\begin{aligned}
& f(\phi, u)= \\
& {\left[\begin{array}{c}
-K_{x g i}\left(\phi_{1}(0)+G_{r e f}\right)\left(\phi_{2}(0)+I_{r e f}\right)+\frac{T_{g h}}{V_{G}} \\
-K_{x i}\left(\phi_{2}(0)+I_{r e f}\right) \\
+\frac{T_{i G \max }}{V_{I}} h\left(\phi_{1}\left(-\tau_{g}\right)+G_{r e f}\right)+v_{r e f}+u
\end{array}\right]}
\end{aligned}
$$

If $\phi \in \mathcal{C}, u \in U$, in (9), are replaced with $x_{t} \in \mathcal{C}, u(t) \in U$, where (see notations) $x_{t}(\tau)=x(t+\tau)=\left[\begin{array}{l}x_{1}(t+\tau) \\ x_{2}(t+\tau)\end{array}\right]$, $\tau \in\left[-\tau_{g}, 0\right]$, then system (8) is equivalent to system (1), with $f$ as in (9). The initial condition $x_{0} \in \mathcal{C}$, for the glucoseinsulin system written in the form of (1), is given by $x_{0}(\tau)=$ $\left[\begin{array}{c}G_{0}-G_{r e f} \\ I_{0}-I_{r e f}\end{array}\right], \tau \in\left[-\tau_{g}, 0\right]$.

Let $\Psi: R^{2} \rightarrow R^{2}$ be defined, for $x=\left[\begin{array}{l}x_{1} \\ x_{2}\end{array}\right] \in R^{2}$, as

$$
\Psi(x)=\left[\begin{array}{c}
x_{1} \\
-K_{x g i}\left(x_{1}+G_{r e f}\right)\left(x_{2}+I_{r e f}\right)+\frac{T_{g h}}{V_{G}}
\end{array}\right] .
$$

The state feedback $\bar{k}$ in forthcoming Theorem 2 has been designed in a continuous time basis in [28], by means of the change of variables in (10).

Theorem 2: Let $\bar{k}: \mathcal{C} \rightarrow R$ be defined, for $\phi \in \mathcal{C}$, as

$$
\begin{gathered}
\bar{k}(\phi)=-v_{r e f}+\frac{1}{K_{x g i}\left(\phi_{1}(0)+G_{r e f}\right)} . \\
\left(\mathcal{P}\left(\phi_{1}(0)+G_{r e f}, \phi_{2}(0)+I_{r e f}, \phi_{1}\left(-\tau_{g}\right)+G_{r e f}\right)\right. \\
-\Gamma \Psi(\phi(0))), \quad \phi_{1}(0) \neq-G_{r e f}, \\
\quad \bar{k}(\phi)=-v_{r e f}, \quad \phi_{1}(0)=-G_{r e f},
\end{gathered}
$$

where $\mathcal{P}: R^{3} \rightarrow R$ is defined, for $y=\left[\begin{array}{lll}y_{1} & y_{2} & y_{3}\end{array}\right]^{T} \in$ $R^{3}$ as

$$
\begin{aligned}
& \mathcal{P}\left(y_{1}, y_{2}, y_{3}\right)=-K_{x g i} y_{2}\left(-K_{x g i} y_{1} y_{2}+\frac{T_{g h}}{V_{G}}\right) \\
& -K_{x g i} y_{1}\left(-K_{x i} y_{2}+\frac{T_{i G m a x}}{V_{I}} h\left(y_{3}\right)\right)
\end{aligned}
$$

and the matrix

$$
\Gamma=\left[\begin{array}{ll}
\Gamma_{1} & \Gamma_{2}
\end{array}\right] \in R^{1 \times 2},
$$

$\Gamma_{i} \in R, i=1,2$, is designed to ensure that the matrix

$$
H=\left[\begin{array}{ll}
0 & 1 \\
0 & 0
\end{array}\right]+\left[\begin{array}{l}
0 \\
1
\end{array}\right] \Gamma
$$

is Hurwitz with (any) prescribed eigenvalues in the left half complex plane. Let $k: \mathcal{C} \rightarrow U$ be defined, for $\phi \in \mathcal{C}$, as

$$
k(\phi)=\left\{\begin{array}{cc}
\bar{k}(\phi), & \bar{k}(\phi) \in U, \\
v_{\text {max }}-v_{\text {ref }}, & \bar{k}(\phi)>v_{\text {max }}-v_{\text {ref }}, \\
-v_{\text {ref }}, & \bar{k}(\phi)<-v_{\text {ref }}
\end{array}\right.
$$


Then, there exists a positive real $Q$ such that the feedback $k$ stabilizes, in the sample-and-hold sense, in $\mathcal{C}_{Q}$ (i.e., locally), the system described by (8) (see Definition 2).

Proof: Let $\Omega_{x}=\left\{x \in R^{2}:|x|<\frac{1}{2} \min \left\{G_{\text {ref }}, I_{\text {ref }}\right\}\right\}$. Let $\Omega_{z}$ be an open neighborhood of the origin such that the map $\bar{\Psi}: \Omega_{x} \rightarrow \Omega_{z}$ defined, for $x \in \Omega_{x}$, as

$$
\bar{\Psi}(x)=\Psi(x)
$$

is a diffeomorphism. The feedback $\bar{k}$ is zero at zero. Indeed, from (7), the following equalities hold

$$
\begin{aligned}
& \frac{\mathcal{P}\left(G_{r e f}, I_{r e f}, G_{r e f}\right)}{K_{x g i} G_{r e f}}=\frac{1}{K_{x g i} G_{r e f}} \cdot \\
& \left(-K_{x g i} I_{r e f}\left(-K_{x g i} G_{r e f} I_{r e f}+\frac{T_{g h}}{V_{G}}\right)\right. \\
& \left.-K_{x g i} G_{r e f}\left(-K_{x i} I_{r e f}+\frac{T_{i G m a x}}{V_{I}} h\left(G_{r e f}\right)\right)\right)= \\
& \frac{1}{K_{x g i} G_{r e f}}\left(K_{x g i} G_{r e f} v_{r e f}\right)=v_{r e f}
\end{aligned}
$$

From (11), (17), taking into account that $\Psi(0)=0$, it follows that $\bar{k}(0)=0$. The feedback $\bar{k}$ is continuous in an open neighborhood of the origin. It follows from (11) that there exists a positive real $\bar{S}$ such that, for all $\phi \in \mathcal{C}_{\bar{S}}, \bar{k}(\phi) \in U$. Thus, in $\mathcal{C}_{\bar{S}}, k(\phi)=\bar{k}(\phi)$ (i.e., input saturation constraints are avoided). Let $S=\min \left\{\bar{S}, \frac{1}{3} \min \left\{G_{\text {ref }}, I_{\text {ref }}\right\}\right\}$. We have to show that the change of coordinates $\bar{\Psi}$ and the feedback $k$ satisfy the hypotheses of Theorem 1 in $\mathcal{C}_{S}$, for this chosen positive real $S$. The relation $B_{S} \cap \Omega_{x}=B_{S}$ holds. As far as inequalities (4) are concerned, we have that the function $x \rightarrow$ $|\psi(x)|, x \in R^{2}$ (see (10)), is positive definite and radially unbounded. Therefore, from Lemma 4.3, p. 145, in [14], it follows that there exist two functions $\underline{\gamma}_{\Psi}$ and $\bar{\gamma}_{\Psi}$, of class $\mathcal{K}_{\infty}$, such that the inequalities (4) hold satisfied. As far as the equality (5) is concerned, it is sufficient to consider (see [8], [9], [21], [24], [29]) that, in the new variables $z=\psi(x)$, $x \in \Omega_{x}$, the chosen feedback, if applied in continuous time, linearizes and stabilizes the system at hand. In details, for any $\phi=\left[\begin{array}{l}\phi_{1} \\ \phi_{2}\end{array}\right] \in \mathcal{C}_{S}, \phi_{i}(\tau) \in R, \tau \in\left[-\tau_{g}, 0\right]$, from (9), (10), (11), (13), (14), (16), we have

$$
\begin{aligned}
& \left.\frac{\partial \bar{\Psi}(x)}{\partial x}\right|_{x=\phi(0)} f(\phi, k(\phi))= \\
& {\left[\begin{array}{cc}
1 & 0 \\
-K_{x g i}\left(\phi_{2}(0)+I_{r e f}\right) & -K_{x g i}\left(\phi_{1}(0)+G_{r e f}\right)
\end{array}\right] .} \\
& f(\phi, k(\phi))=
\end{aligned}
$$

$$
\left[\begin{array}{c}
-K_{x g i}\left(\phi_{1}(0)+G_{r e f}\right)\left(\phi_{2}(0)+I_{r e f}\right)+\frac{T_{g h}}{V_{G}} \\
-K_{x g i}\left(\phi_{2}(0)+I_{r e f}\right) \cdot \\
\left(-K_{x g i}\left(\phi_{1}(0)+G_{r e f}\right)\left(\phi_{2}(0)+I_{r e f}\right)+\frac{T_{g h}}{V_{G}}\right) \\
-K_{x g i}\left(\phi_{1}(0)+G_{r e f}\right) \cdot \\
\left(-K_{x i}\left(\phi_{2}(0)+I_{r e f}\right)\right. \\
+\frac{T_{i G m a x}}{\left.V_{I}\left(\phi_{1}\left(-\tau_{g}\right)+G_{r e f}\right)+v_{r e f}\right)}-K_{x g i}\left(\phi_{1}(0)+G_{r e f}\right) \cdot \\
\left(-v_{r e f}\right. \\
+\frac{\mathcal{P}\left(\phi_{1}(0)+G_{r e f}, \phi_{2}(0)+I_{r e f}, \phi_{1}\left(-\tau_{g}\right)+G_{r e f}\right)-\Gamma \Psi(\phi(0))}{K_{x g i}\left(\phi_{1}(0)+G_{r e f}\right)}
\end{array}\right]=
$$

Since the matrix $H$ in (14) is Hurwitz, the hypotheses of Theorem 1 are satisfied. It follows that there exists a positive real $Q$ such that the (bounded) state feedback $k$ stabilizes in the sample-and-hold sense, in $\mathcal{C}_{Q}$, the glucose-insulin system described by (8), which describes the deviation from the reference value of glucose and insulin. We conclude that, if the initial value of the glucose and the insulin are sufficiently near $\left(G_{r e f}, I_{r e f}\right)$, then the feedback $k$ yields stabilization in the sample-and-hold sense of the system described by (6), in a neighborhood of $\left(G_{\text {ref }}, I_{\text {ref }}\right)$.

According to (11), (15), recalling the relation $u(t)=$ $v(t)-v_{r e f}$, the piece-wise constant control law $v(t)$ in (6) is defined as follows, for $t \geq 0$,

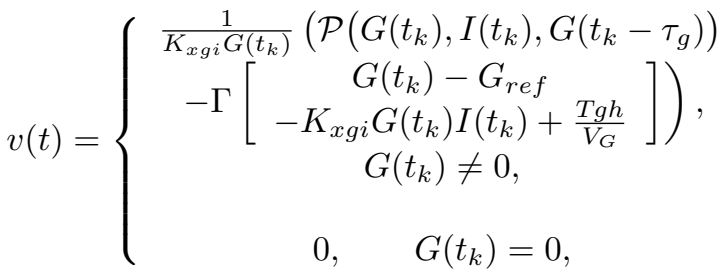

$$
\begin{aligned}
& t_{k} \leq t \leq t_{(k+1)}, k=0,1, \ldots, t_{0}=0,
\end{aligned}
$$

with $\mathcal{P}$ defined in (12). If the right-hand side of (19) exceeds $v_{\max }$ or is negative, then it is set $v(t)=v_{\max }$ or $v(t)=0$, respectively. At this stage, such cases cannot be a-priori excluded, though Theorem 1 ensures that, if the sampling period is chosen suitably small and the initial values of glucose and insulin are sufficiently near the desired values $G_{r e f}, I_{r e f}$, the saturation constraints are avoided. Indeed, here just the existence of a suitable region of attraction $\mathcal{C}_{Q}$ and of a suitable small sampling period is proved, for 
TABLE I

GLUCOSE-INSULIN SYSTEM PARAMETERS

\begin{tabular}{|l|l|}
\hline$G_{b}$ & $10.37 \mathrm{mM}$ \\
$\gamma$ & 3.205 \\
$V_{G}$ & $0.187 \mathrm{~L} / \mathrm{kgBW}$ \\
$V_{I}$ & $0.25 \mathrm{~L} / \mathrm{kgBW}$ \\
$I_{b}$ & $48.95 \mathrm{pM}$ \\
$G^{\star}$ & $9 \mathrm{mM}$ \\
$K_{x i}$ & $1.211 \mathrm{e}-2 \mathrm{~min}^{-1}$ \\
$K_{x g i}$ & $3.11 \mathrm{e}-5 \mathrm{~min}^{-1} \mathrm{pM}^{-1}$ \\
$T_{i G m a x}$ & $0.242 \mathrm{~min}^{-1} \mathrm{pmol} / \mathrm{kgBW}$ \\
$\tau_{g}$ & $24 \mathrm{~min}^{-1} \mathrm{mmol} / \mathrm{kgBW}$ \\
$T_{g h}$ & $0.003 \mathrm{~min}^{-1} \mathrm{~mm}^{2}$ \\
\hline
\end{tabular}

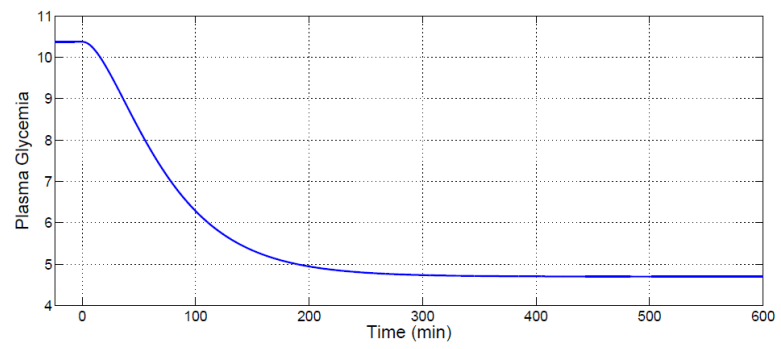

Fig. 1. Evolution of the plasma glycemia $G(t)$, [mM], with sampling period $\delta=10 \mathrm{~min}$

the stabilization in the sample-and-hold sense. An analysis of the region of attraction $Q$, as well as of the required suitably small sampling period, are not studied here and are left for future work. Theorem 5.3, 5.5, and their proofs, in [32], provide some insights for this further theoretical investigation.

\section{Simulations}

We considered, for simulation, the case 3 (severe hyperglycemia, establishment of a state of frank Type 2 Diabetes Mellitus) reported in [28] (see Table I, where $G_{b}$ and $I_{b}$ denote steady state values of glycemia and insulinemia when no control action is taken, i.e., when $v(t) \equiv 0, t \in R^{+}$). As far as the eigenvalues for the matrix $H$ in (14) are concerned, they are chosen equal to $-0.02,-0.03$. The desired level of glycemia is set at $G_{r e f}=4.7 \mathrm{mM}$. The initial value of glycemia $G_{0}$ and initial value of insulinemia $I_{0}$ are set equal to the (input-free) steady state values $G_{b}$ and $I_{b}$, respectively. Simulation results are reported for the sampling period $\delta$ set

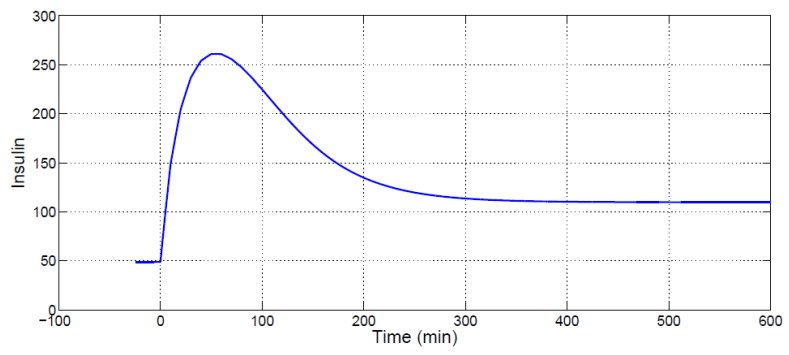

Fig. 2. Evolution of the insulin $I(t),[\mathrm{pM}]$, with sampling period $\delta=$ $10 \mathrm{~min}$

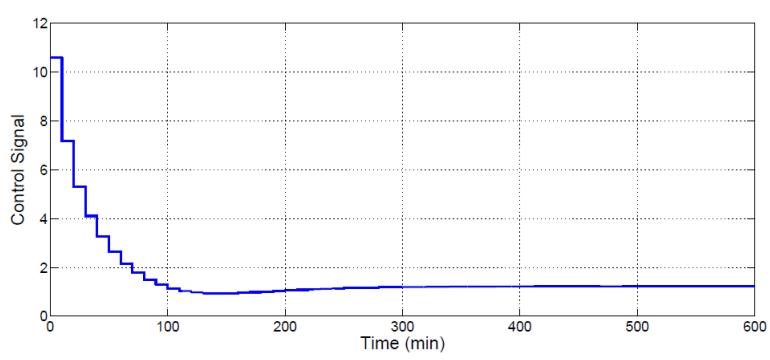

Fig. 3. Control Signal $v(t),[\mathrm{pM} / \mathrm{min}]$, with sampling period $\delta=10 \mathrm{~min}$

equal to $10 \mathrm{~min}$. With sampling period equal to $120 \mathrm{~min}$, saturation constraints and dangerous oscillations appear, and the controller does not provide acceptable performances. In the case of $\delta=60 \mathrm{~min}$, saturations constraints appear too. Indeed the controller (19) provides infeasible negative values for the control signal. By imposing in the simulations that the control law is equal to 0 whenever the controller provides a negative control law, the plasma glycemia is driven to a sufficiently small neighborhood of the desired value. If the sampling period is chosen equal to $30 \mathrm{~min}$, saturations problem do not appear any more and the plasma glycemia is driven to the desired value in a good fashion, without dangerous oscillations. By choosing the sampling period equal to $10 \mathrm{~min}$, the behavior of the plasma glycemia and of the insulin are reported in Figs. 1 and in Fig. 2, respectively. The input signal is reported in Fig. 3. The plasma glycemia is driven to the desired level, in an excellent fashion without oscillations. The piece-wise constant control law never becomes negative. Practically the same results are obtained with sampling period equal to 5 min.

Remark 1: The simulation results here shown for the case of $\delta=10 \mathrm{~min}$, for the glucose-insulin system (6), are well known in the literature (see [29], where an observer to avoid insulin measures is even exploited), and are reported here only for reader's convenience, in order to validate the theoretical results here provided. A theoretical proof of the (local) convergence of the glucose variable to (an arbitrarily small neighborhood of) the reference value, by means of the sampled-data controller (19), though well known in the literature from the many performed simulations, was missing so far. The algorithm here provided has been validated by simulations in closed loop with standard computer simulators for the artifical pancreas (see [17], [30]). The theoretical proof here provided adds a further property to the controller proposed first in [28] and improved by the use of observers in [29].

\section{Conclusions}

A stabilizing continuous-time controller proposed in the literature for the regulation of plasma-glycemia has been here proved to be a local stabilizer in the sample-and-hold sense. This theoretical proof adds an important property to a controller which has been shown in the literature to perform very well in closed loop with standard computers simulators of human glucose-insulin system. An interesting topic to 
be investigated concerns the stabilization in the sample-andhold sense of the glucose-insulin system, by means of the sampled-data implementation of the observer-based (continuous time) controller proposed in [29], which allows to avoid insulin measures. A theoretical proof of the stabilization in the sample-and-hold sense when the above controller is implemented by digital devices, as standard in practice, is not yet available in the literature. The many simulations performed in [29], with a sampled-data implementation of the observer-based state feedback, encourage to look for this (expected, not yet proved) theoretical result.

A further theoretical investigation will concern the possibility of achieving stabilization in the sample-and-hold sense, by means of a sampled-data state feedback which makes use of measurements not at the current sampling time, but (at least) at the previous one. This theoretical result would allow to use more trustworthy glucose measurements for actual glucose control therapies, and would lay the foundations, in the future, for an artificial pancreas exploiting insulin measurements as well, known to be much more cumbersome to obtain in real-time.

\section{REFERENCES}

[1] F. Chee, T. Fernado, "Closed-Loop Control of Blood Glucose", Springer, 2007.

[2] F.H. Clarke, "Discontinuous Feedback and Nonlinear Systems", Plenary Lecture at IFAC Conference on Nonlinear Control Systems (NOLCOS), Bologna, Italy, 2010, IFAC-PapersOnline.

[3] F.H. Clarke, Y.S. Ledyaev, E.D. Sontag, A.I. Subbotin, "Asymptotic controllability implies feedback stabilization," IEEE Trans. on Automatic Control, Vol. 42, pp. 1394-1407, 1997.

[4] P. Dua, F.J. Doyle, E.N. Pistikopoulos, "Model-based blood glucose control for Type 1 diabetes via parametric programming", IEEE Trans. Biomed. Eng., Vol. 53, pp. 1478-1491, 2006.

[5] E. Fridman, "Introduction to Time-Delay Systems: Analysis and Control", Birkhauser, Basel, 2014.

[6] E. Fridman, "A refined input delay approach to sampled-data control", Automatica, Vol. 46, N. 2, pp. 421-427, 2010.

[7] E. Fridman, A. Seuret, J.-P. Richard, "Robust sampled-data stabilization of linear systems: and input delay approach", Automatica, Vol. 40, pp. 1441-1446, 2004.

[8] A. Germani, C. Manes, and P. Pepe, "Local asymptotic stability for nonlinear state feedback delay systems," Kybernetika, Vol. 36, No. 1, pp. 31-42, 2000.

[9] A. Germani, C. Manes, and P. Pepe, "Input-output linearization with delay cancellation for nonlinear delay systems: the problem of the internal stability," International Journal of Robust and Nonlinear Control, Vol. 13, No. 9, pp. 909-937, 2003.

[10] L. Grune and D. Nesic, "Optimization based stabilization of sampleddata nonlinear systems via their approximate discrete-time models," SIAM Journal on Control and Optimization, vol. 42, pp. 98-122, 2003.

[11] J. K. Hale, and S. M. Verduyn Lunel, "Introduction to Functional Differential Equations", Springer Verlag, 1993.

[12] R. Hovorka, V. Canonico, L.J. Chassin, U. Haueter, M. MassiBenedetti, M.O. Federici, T.R. Pieber, H.C. Schaller, L. Schaupp, T. Vering, M.E. Wilinska, "Nonlinear model predictive control of glucose concentration in subjects with type I diabetes, Physiol. Measure., Vol. 25, pp. 905-920, 2004.

[13] I. Karafyllis, M. Krstic, "Nonlinear Stabilization Under Sampled and Delayed Measurements, and with Inputs Subject to Delay and ZeroOrder Hold", IEEE Transactions on Automatic Control, Vol. 57, N. 5, pp. 1141-1154, 2012.

[14] H. K. Khalil, "Nonlinear Systems", Prentice Hall, International Edition, Third Edition, Upper Saddle River, New Jersey, 2000.

[15] V. Kolmanovskii and A. Myshkis, "Introduction to the theory and applications of functional differential equations", Kluwer Academic Publishers, Dordrecht, 1999.
[16] L. Kovacs, P. Szalay, Z. Almassy, L. Barkai, "Applicability Results of a Non- linear Model-Based Robust Blood Glucose Control Algorithm", Journal of Diabetes Science and Technology, 7(3), pp. 708-716, 2013.

[17] B.P. Kovatchev, M.D. Breton, C. Dalla Man and C. Cobelli, "In silico model and computer simulation environment approximating the human glucose/insulin utilization", Food and Drug Administration Master File MAF 1521, 2008.

[18] D.S. Laila, D. Nesic, A. Teel, "Open- and Closed-Loop Dissipation Inequalities Under Sampling and Controller Emulation", European Journal of Control, N. 8, pp. 109-125, 2002.

[19] K. Liu, E. Fridman, "Wirtinger's inequality and Lyapunov-based sampled-data stabilization", Automatica, 48(1), pp. 102-108, 2012.

[20] L. Magni, D.M. Raimondo, C. Dalla Man, G. De Nicolao, B. Kovatchev, C. Cobelli, "Model predictive control of glucose concentration in type I diabetic patients: an in silico trial", Biomed. Signal Process. Control, Vol. 4, pp. 338-346, 2009.

[21] L.A. Marquez-Martinez, and C.H. Moog, "Input-output feedback linearization of time-delay systems", IEEE Transactions on Automatic Control, Vol. 49, No. 5, pp. 781-785, 2004.

[22] D. Nesic, L. Grune, "Lyapunov-based continuous-time nonlinear controller redesign for sampled-data implementation", Automatica, Vol. 41, pp. 1143-1156, 2005.

[23] D. Nesic and A. Teel, "A framework for stabilization of nonlinear sampled- data systems based on their approximate discrete-time models," IEEE Trans. on Automatic Control, 49(7), pp. 1103-1122, 2004.

[24] T. Oguchi, A. Watanabe and T. Nakamizo, "Input-output linearization of retarded non-linear systems by using an extension of Lie derivative", International Journal of Control, Vol. 75, No. 8, 582-590, 2002.

[25] H. Omran, L. Hetel, J.-P. Richard, F. Lamnabhi-Lagarrigue, "Stability analysis of bilinear systems under aperiodic sampled-data control", Automatica, Vol. 50, pp. 1288-1295, 2014.

[26] P. Palumbo, S. Panunzi and A. De Gaetano, "Qualitative behavior of a family of delay differential models of the glucose insulin system", Discrete and Continuous Dynam. Systems - B, Vol. 7, pp. 399-424, 2007.

[27] P. Palumbo, P. Pepe, S. Panunzi, A. De Gaetano, "Digital Closed-Loop Control of Plasma Glycemia", $49^{\text {th }}$ IEEE Conference on Decision and Control, Atlanta, USA, pp. 833-838, 2010.

[28] P. Palumbo, P. Pepe, S. Panunzi, A. De Gaetano, "Robust Closed-Loop Control of Plasma Glycemia: a Discrete Delay Model Approach", Discrete and Continuous Dynamical Systems - Series B, Vol. 12, No. 2, pp. 455-468, 2009.

[29] P. Palumbo, P. Pepe, S. Panunzi, A. De Gaetano, "Time-delay modelbased control of the glucose-insulin system, by means of a state observer", European Journal of Control, Vol. 18, N. 6, pp. 591-606, 2012.

[30] P. Palumbo, G. Pizzichelli, S. Panunzi, P. Pepe, A. De Gaetano, "Model-based control of plasma glycemia: tests on populations of virtual patients", Mathematical Biosciences, Vol. 257, pp. 2-10, 2014.

[31] S. Panunzi, P. Palumbo and A. De Gaetano "A discrete single delay model for the Intra-Venous Glucose Tolerance Test", Theoretical Biology and Medical Modelling, Vol. 4, No.35, pp. 1-16, 2007.

[32] P. Pepe, "Stabilization in the Sample-and-Hold Sense of Nonlinear Retarded Systems", SIAM Journal on Control and Optimization, Vol. 52, N. 5, pp. 3053-3077, 2014.

[33] P. Pepe, "Robustification of nonlinear stabilizers in the sample-andhold sense", Journal of The Franklin Institute, Vol. 352, pp. 41074128, 2015.

[34] P. Pepe, "On stability preservation under sampling and approximation of feedbacks for retarded systems", SIAM Journal on Control and Optimization, Vol. 54, N. 4, pp. 1895-1918, 2016.

[35] C. Prieur, "Asymptotic Controllability and Robust Asymptotic Stabilizability", SIAM Journal on Control and Optimization, Vol. 43, N. 5, pp. 1888-1912, 2005.

[36] C. Prieur, E. Trélat, "Quasi-optimal robust stabilization of control systems", SIAM Journal on Control and Optimization, Vol. 45, N. 5, pp. 1875-1897, 2006.

[37] A. Seuret, "A novel stability analysis of linear systems under asynchronous samplings", Automatica, Vol. 48, pp. 177-182, 2012.

[38] A. Seuret, M.M. Peet, "Stability Analysis of Sampled-Data Systems Using Sum of Squares", IEEE Transactions on Automatic Control, Vol. 58, N. 6, pp. 1620-1625, 2013.

[39] E.D. Sontag, "Clocks and insensitivity to small measurement errors", ESAIM Control, Optimisation and Calculus of Variations, Vol. 4, pp. 537-557, 1999. 